\title{
Blood Microsampling for Complete Blood Count: Take Heed of Preanalytical Errors
}

\author{
Barbara Kościelniak ${ }^{1}$, Andrzej Zając ${ }^{2}$ and Przemysław Tomasik ${ }^{1 *}$ \\ ${ }^{1}$ Department of Clinical Biochemistry, Jagiellonian University Medical College, Cracow, Poland \\ ${ }^{2}$ Departament of Pediatric Surgery, University Children Hospital, Cracow, Poland
}

\begin{abstract}
Background: The credibility of the result of a complete blood count is closely connected with the preanalytical phase.

Objectives: This study evaluated accordance of filling of microtubes with manufacturer's recommendation and assessed the effect of storage of overfilled and underfilled samples on the results of complete blood count.

Design and Methods: Volume of blood samples collected into microtubes in the wards of the University Children's Hospital in Cracow during one month was analyzed. In the stability studies, overfilled and underfilled samples stored at ambient temperature were analyzed at 1,2, 3 and 12 hours after phlebotomy. The analysis was made using the SYSMEX XT-1800i analyzer.

Results: More than half of the analyzed samples were incorrectly filled. $63 \%$ of the samples were filled above the manufacturer's recommended volume and $15 \%$ of test-tubes were filled below the recommendation. We observed differences between collected blood volume in accordance to the age of patients $(p=0.001)$. The storage of overfilled and underfilled microtubes for complete blood count for $1,2,3$ and $12 \mathrm{~h}$ at room temperature had no effect on the results of this test.

Conclusion: Medical staff does not follow the instructions of the manufacturers. It might lead to a decrease of the quality and credibility of the results.
\end{abstract}

Keywords: Phlebotomy, preanalytical error, nurse training, children.

\section{INTRODUCTION}

Complete blood count (CBC) is one of the basic health screening and target tests performed in the diagnostic laboratories. The effective and trustworthy laboratory service is the amalgamation of accuracy, precision, and rapidity of results delivered to the clinicians as well as to the patients. Notwithstanding dynamic progress in laboratory science, it is still susceptible to various errors classified as preanalytical, analytical, and postanalytical. The majority of errors affecting laboratory analysis occur in the preanalytical phase, mainly because of the difficulty in achieving standardized procedures for sample collection $[1,2]$. It includes improper preparation of the patient, wrong collection procedure, incorrect transport, preparation and labeling of the samples $[3,4]$. In the case of CBC measurement, one of the requirements is that blood must fill the tube to volume recommended by the manufacturer and adequate blood to anticoagulant ratio preserved $[3,5]$. It was shown in previous studies that improper filling of microtubes results of the impossibility of analysis in the case of significant underfilling and false results in the case of significant overfilling [6].

*Address correspondence to this author at the Department of Clinical Biochemistry, University Children's Hospital, Jagiellonian University, Medical Collegue, Wielicka St 265, 30-663 Cracow, Poland; Tel: +48126582011;

Fax: +48126580681; E-mail: p.tomasik@uj.edu.pl
The aim of the study was to assess the accordance of the volume of blood samples drawn for laboratory testing in pediatric wards with recommendations of the manufacturer as well as with the hospital standard operating procedures (SOPs). Moreover, we analyzed the influence of time of blood storage in microtubes on the $\mathrm{CBC}$ results.

\section{MATERIALS AND METHODS}

In order to evaluate the volume of blood collected into the microtubes in the Children's University Hospital, 1485 microsamples provided to the laboratory from $1^{\text {st }}$ March 2016 to $30^{\text {th }}$ March 2016, were analyzed. The capillary blood samples were collected in the wards by nurses. The sanitized child's finger was punctured using quick and smooth motion. Then blood was collected in open system using capillary connected

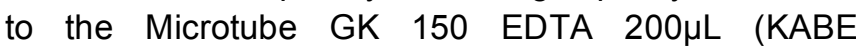
Labortechnik GmbH, Nümbrecht - Elsenroch, Germany). When the blood filled microtube, the capillary was dismantled, microtube closed and blood was mixed. The blood volume in each microtube was evaluated by comparing to series of standard volumes. Volume standards ranged from $20 \mu \mathrm{L}$ to $900 \mu \mathrm{L}$ (with a difference of $20 \mu \mathrm{L}$ between particular samples). Laboratory Information System (LIS) was used to collect information on patient's age, sex, and the hospital ward where the patient had the blood 
collected. The next step was to estimate if the blood storage of underfilled and overfilled KABE GK 150 EDTA $200 \mu \mathrm{L}$ samples may affect the CBC test values. Blood was collected from 30 healthy, adult volunteers (80\% men) with age median 29 (26-30) years into series of microtubes. From each patient, six microtubes were filled with $700 \mu \mathrm{L}$ and 6 microtubes were filled with $120 \mu \mathrm{L}$ (minimal blood volume required by the analyzer for test performing) at the same time. In each microtube the CBC parameters were examined immediately after sampling, and after 1, 2, 3, and $12 \mathrm{~h}$ of storage at ambient temperature. The analysis was performed using hematological analyzer SYSMEX XT$1800 i$.

The Jagiellonian University Ethical Committee approved the study, and all the volunteers provided their written informed consent.

\section{Statistic}

Continuous variables were presented as a median and interquartile range (IQR) or as a mean \pm standard deviation and categorical variables were presented as numbers (percentages). Quantitative variables were checked for the normality of their distribution using the Shapiro-Wilk test. In order to evaluate differences between various blood volume collected into microtubes and to estimate if the blood storage in overfilled tubes may affect the CBC test values, Kruskal-Wallis one-way analysis of variance by ranks for non-normally distributed continuous variables were performed together with posthoc Dunn's test when necessary. Results were considered statistically significant at $p$ values less than 0.05 . Statistical analysis was performed using STATISTICA v.10, (StatSoft Inc., USA).

\section{RESULTS}

\section{The Volume of Blood Collected in the Wards of the} University Children's Hospital in Cracow

The median of blood volume, which was collected

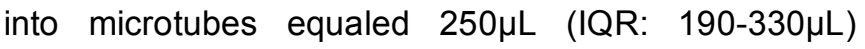
whereas the recommended by manufacturer blood filling of KABE microtubes is $200 \mu \mathrm{L} \pm 10 \%$. Only 335 microtubes $(22 \%)$ were filled in accordance with the manufacturer's instructions $(180-220 \mu \mathrm{L})$, while 930 $(63 \%)$ of the samples were filled above the manufacturer's recommended volume and $228(15 \%)$ of test-tubes were filled below the recommended blood volume (Figure 1).

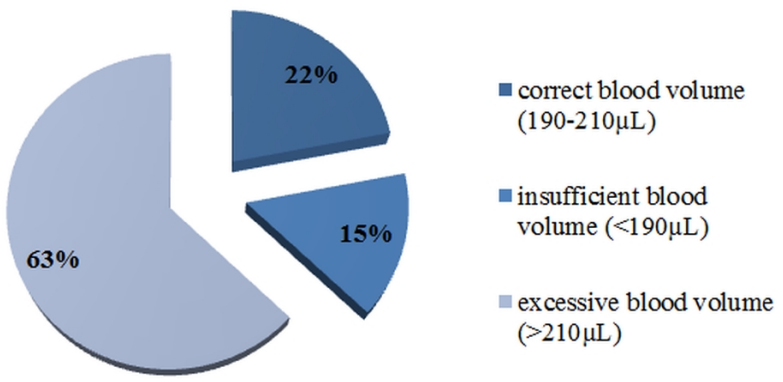

Figure 1: The amount of blood collected into microtubes in hospital wards.

The median age of the patients was 5.6 years (IQR: 1.5-11 years). The percentage of girls was $45 \%$, while boys constituted $55 \%$. We did not observe any differences between blood volume collected on hospital wards with regard to gender $(p=0.176)$.

We noted that in the neonatal wards and in the operating room, the collected volume of blood was mostly underfilled, and the average blood volume was $130 \mu \mathrm{L}$. The highest volumes of blood were drown in gastro-enterology ward - the average blood volume was $440 \mu \mathrm{L}$. We observed also the tendency to collection more blood volume from older children $(p=0.001)$. Moreover, we noted that the medical staff collected excessive blood volume more frequently from older chiIdren when compared to correct blood volume $(p=0.001)$ and simultaneously they collected insufficient blood volume from neonates and infants $(p=0.006$; Figure 2$)$.

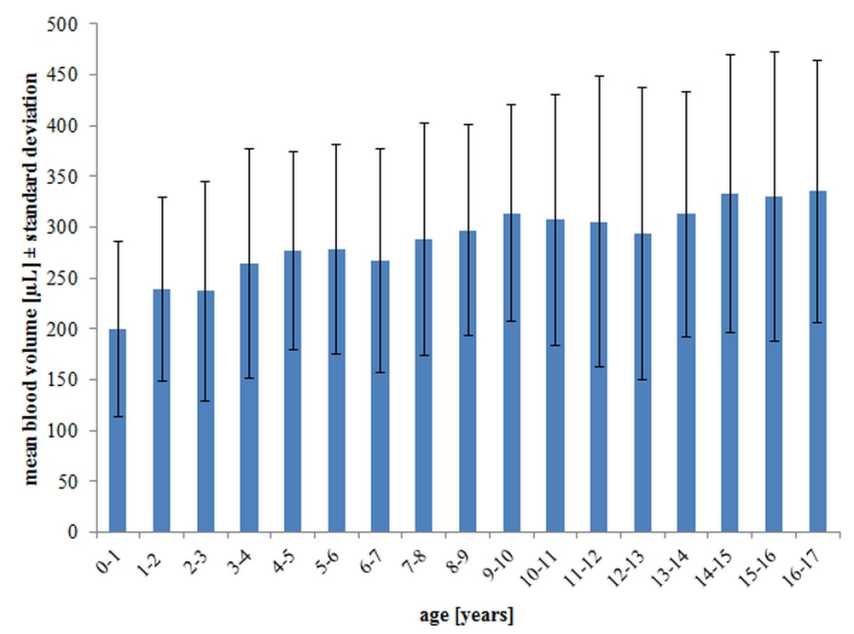

Figure 2: The relationship between patients' age and the blood volume median.

\section{The Assessment of the Impact of Storage Time on the Results of Complete Blood Counts}

We have not noted any significant differences in the number of erythrocytes (RBC), platelets (PLT), white blood cells (WBC), concentration of hemoglobin (HGB), 
values of hematocrit (HTC), mean cell volume (MCV), mean corpuscular hemoglobin $(\mathrm{MCH})$, and mean corpuscular hemoglobin concentration (MCHC) (Figures 3, 4) depending on the time of blood stored at microtubes at room temperature up to $12 \mathrm{~h}$ (all cases $p>0.05$ ). Moreover, we have not found any significant changes in the results of $\mathrm{CBC}$ obtained in underfilled tubes after storage for 1, 2, 3 and $12 \mathrm{~h}$ after phlebotomy in ambient temperature (all cases $p>0.05$ ).

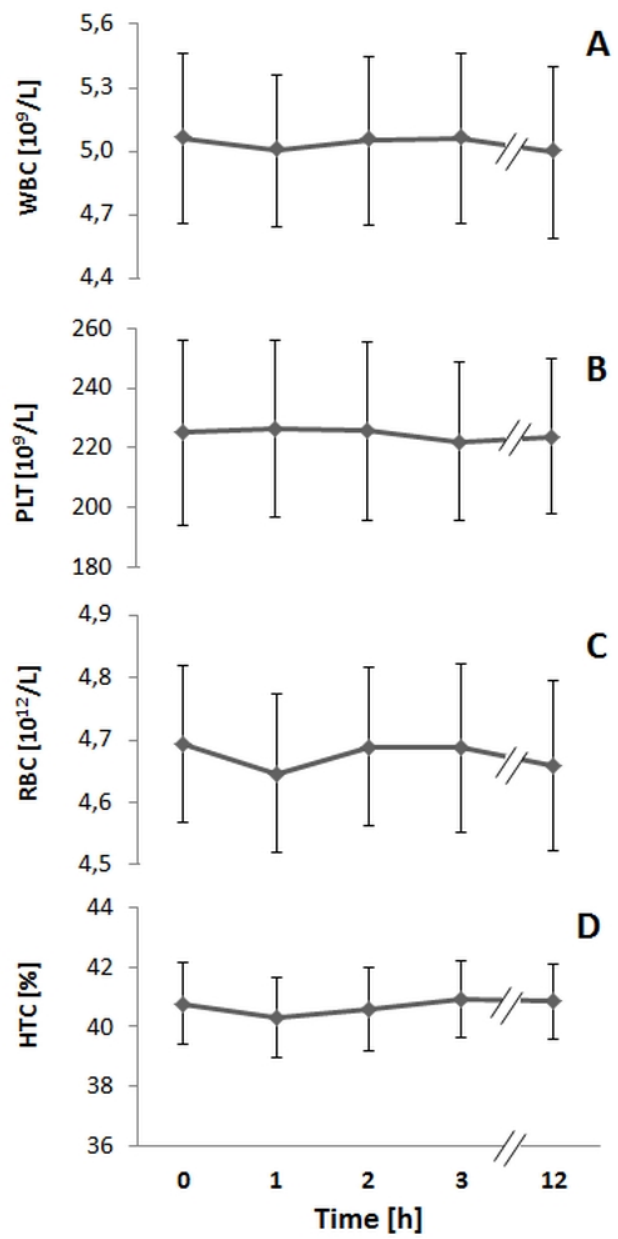

Figure 3: The impact of storage time on the results of WBC, PLT, RBC and HTC.

Abbreviations: WBC, white blood cells; PLT, platelets; RBC, red blood cells and HTC, hematocrit level.

\section{DISCUSSION}

Blood collection is the most common invasive procedure in healthcare. Previously, the Working group for Preanalytical Phase (WG-PRE) of the European Federation for Clinical Chemistry and Laboratory Medicine (EFLM) has reported that blood sampling in Europe is performed by different professions with significantly different levels of education, background, skills as well as competence [7]. Numerous studies on the preanalytical phase showed that the collecting of
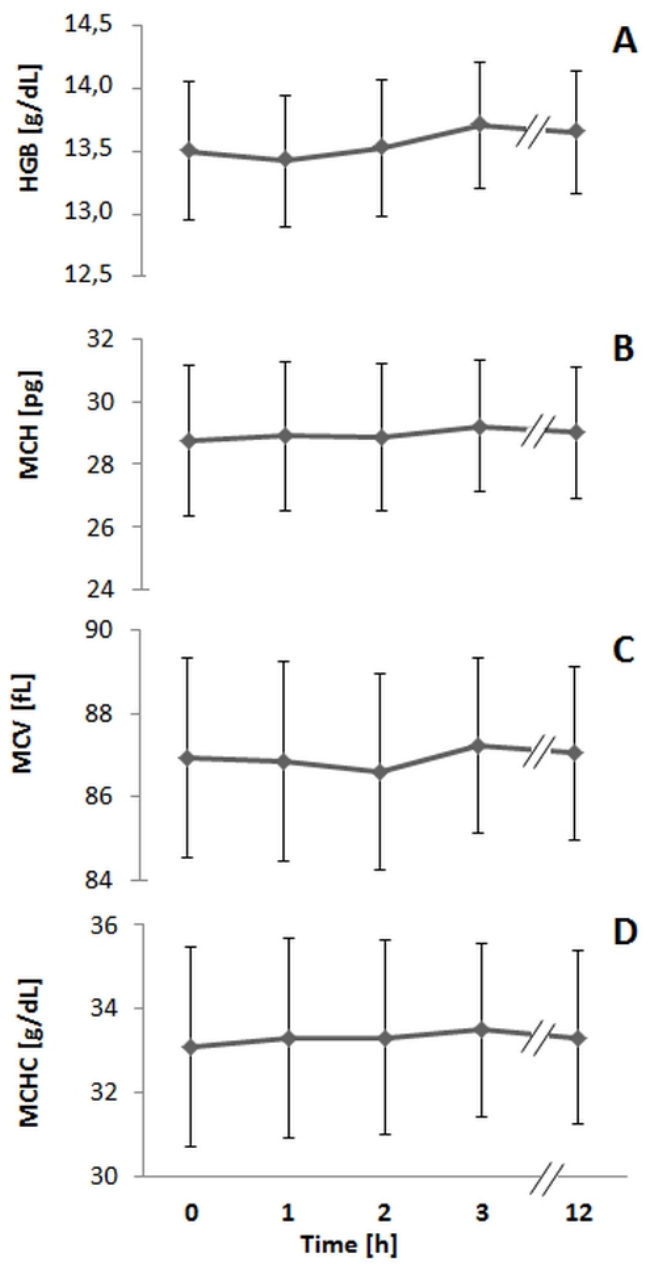

Figure 4: The impact of storage time on the results of $\mathrm{HGB}$, $\mathrm{MCV}, \mathrm{MCH}$ and $\mathrm{MCHC}$.

Abbreviations: HGB, concentration of hemoglobin; MCV, mean cell volume; $\mathrm{MCH}$ mean corpuscular hemoglobin and $\mathrm{MCHC}$, mean corpuscular hemoglobin concentration.

adequate amount of specimen plays a key role in the diagnostic process [8.9.10]. It is a common phenomenon that medical staff does not keep the manufacturer's instructions for filling tubes. Many authors draw attention also to the significant disproportion between blood volume collected from the patient and amount of material necessary to perform test $[11,12,13]$.

Our study has shown that there is the tendency to collect higher blood volume to open system microtubes for CBC testing than recommended by the manufacturer. It was observed mainly in adolescent wards. In our study, we have noted the relationship between blood sampling volume and the age of patients. We hypothesized that because blood loss attributable to laboratory testing is the primary cause of anemia among preterm infants during the first weeks of life, the phlebotomists try to collect the slightest - in 
their opinion - blood volume $[11,14]$. Unfortunately, very often this amount is insufficient for the analysis and the second taking of a blood sample is necessary. On the other hand, the phlebotomist wanting to mitigate patients' pain connected with blood sampling, collect excessive blood volume lest there is a necessity for analysis repetition. Another reason for excessive blood volume collection may be the discrepancy between tube internal volume and nominal filling recommended by a producer. This may mislead phlebotomist. As it was also realized, the common practice in pediatric wards is transferring venous blood from syringes directly to microtubes or fill microtubes with several portions of blood through the integrally mounted capillary.

The use of open systems as KABE microtubes leads also to a greater chance of blood exposure to healthcare workers. Despite these drawbacks, open microsystems are still commonly used for diagnostic phlebotomy in the pediatric population in order to minimize the volume of blood taken from children however in mature patients closed systems for blood sampling are usually used to collect a specimen for the CBC. Their advantage over open systems is minimizing of underfilling or overfilling of the tubes, and always the required sample to anticoagulant ratio is preserved [15].

In the case of $\mathrm{CBC}$ analysis collection errors regarding blood volume using opens systems results in inappropriate anticoagulant to blood ratio [16]. It was shown that the overfilling may lead to the micro-clots formation and to decrease in a number of morphotic elements of blood $[17,18]$. Moreover, in a previous study, we observed that excess collection of blood volume resulted in a decrease of a number of erythrocytes, concentration of hemoglobin, hematocrit and mean corpuscular volume as well as in increase in the number of platelets [6].

The preanalytical error may also be associated with the prolonged time between collection and sample analysis. The data of the present study has shown that storage of overfilled and underfilled samples in microtubes produced by KABE up to 12 hours in ambient condition has no significant effect on results of CBC. Similar results of the study were obtained by de Beca et al. via Sysmex XE-2100 who measured hematological parameters in $\mathrm{K}_{2}$-EDTA-anticoagulated vein blood specimens selected from the routine laboratory workload [19]. They have shown that total WBC, RBC, HGB, and PLT were stable at room temperature for up to 3 days after collection of blood. Also, Vogelaar et al. measured hematological parameters in different storage conditions in EDTA blood collected from 304 apparently healthy individuals aged between 17 and 70 years [20]. They found that stability of samples at room temperature during 2 days was acceptable for morphotic elements of blood such as erythrocytes, lymphocytes, and platelets. The only differential count was changed over time. Probably it is due to change in white blood cells structure which leads to errors in their automated classification. However, the total white blood count (WBC) did not vary.

\section{CONCLUSIONS}

The problem is highly relevant, as changes connected with an improper filling of microtubes with blood have a negative influence on making a definitive medical diagnosis. False test results can lead to incorrect interpretation of patient's state of health. Preanalytic error prevention requires excellent communication and cooperation among all members of the medical personnel - from the clinicians who ordered analysis, to a phlebotomist who collects the specimens, to the laboratory staff receiving the specimen. Quality improvement initiatives should, therefore, take into account mainly preanalytical errors associated with incorrect sample collection. The education of phlebotomy teams can help to reduce those errors. These educational activities should be a continuous part of laboratory quality control procedures because each deviation from the recommended guidelines can relevantly affect the quality of the sample analysis and puts patients health at risk.

\section{DECLARATION OF CONFLICTING INTERESTS}

Hereby, the authors certify that there is no conflict of interest of any kind, which would concern the studies described in the submitted manuscript.

\section{REFERENCES}

[1] Narayanan S. The preanalytic phase. An important component of laboratory medicine. Am J Clin Pathol 2000; 113: 429-452. https://doi.org/10.1309/CONM-Q7R0-LL2E-B3UY

[2] Lippi G, Chance JJ, Church S, Dazzi P, Fontana R, Giavarina $D$ et al. Preanalytical quality improvement: from dream to reality. Clin Chem Lab Med 2011; 49: 1113-1126. https://doi.org/10.1515/CCLM.2011.600

[3] Gilor S, Gilor C. Common laboratory artifacts caused by inappropriate sample collection and transport: how to get the most out of a sample. Top Companion Anim Med 2011; 26: 109-18.

https://doi.org/10.1053/j.tcam.2011.02.003 
[4] Lippi G, Bassi A, Brocco G, Montagnana M, Salvagno GL, Guidi GC. Preanalytic error tracking in a laboratory medicine department: results of a 1-year experience. Clin Chem 2006; 52: $1442-1443$.

https://doi.org/10.1373/clinchem.2006.069534

[5] World Health Organization, Guidelines on Drawing Blood: Best Practices in Phlebotomy Blood-sampling systems. Geneva, Switzerland, 2011.

[6] Kościelniak BK, Kowalczyk PK, Manda-Handzlik AM, Tomasik PJ. The influence of overfilling of microsamples on the results of a Complete Blood Count. Int J Lab Hematol 2015; 37:e141-4

[7] Simundic AM, Church S, Cornes MP, Grankvist K, Lippi G, Nybo $\mathrm{M}$ et al. Compliance of blood sampling procedures with the CLSI H3-A6 guidelines: An observational study by the European Federation of Clinical Chemistry and Laboratory Medicine (EFLM) working group for the preanalytical phase (WG-PRE). Clin Chem Lab Med 2015; 53: 1321-1331. https://doi.org/10.1515/cclm-2014-1053

[8] Adcock DM, Kressin DC, Marlar RA. Minimum specimen volume requirements for routine coagulation testing: dependence on citrate concentration. Am J Clin Pathol 1998; 109: 595-9.

https://doi.org/10.1093/ajcp/109.5.595

[9] Dale JC, Ruby SG. Specimen collection volumes for laboratory tests. Arch Pathol Lab Med 2003; 127: 162-8. https://doi.org/10.1016/j.cca.2015.03.022

[10] Lippi G, Caola I, Cervellin G, Milanesi B, Morandini M, Giavarina D. Error rates during blood collection in emergency departments and outpatient clinics: Results of a prospective multicenter study. Clin Chim Acta 2015; 445: 91-92. https://doi.org/10.1097/PCC.0b013e318219681d

[11] Valentine SL, Bateman ST. Identifying factors to minimize phlebotomy-induced blood loss in the pediatric intensive care unit. Pediatr Crit Care Med 2012; 13: 22-7. https://doi.org/10.1515/cclm-2012-0672
[12] Sztefko K, Beba J, Mamica K, Tomasik P. Blood loss from laboratory diagnostic tests in children. Clin Chem Lab Med 2013; 1: 1-4. https://doi.org/10.1111/j.1365-3148.2009.00960.x

[13] Pabla L, Watkins E, Doughty HA. A study of blood loss from phlebotomy in renal medical inpatients. Transfus Med 2009; 19: 309-14.

[14] Lowe G, Stike R, Pollack M, Bosley J, O'Brien P, Hake A, et al. Nursing blood specimen collection techniques and hemolysis rates in an emergency department: analysis of venipuncture versus intravenous catheter collection techniques. J Emerg Nurs 2008; 34: 26-32. https://doi.org/10.1016/i.jen.2007.02.006

[15] Rana SV. No Preanalytical Errors in Laboratory Testing: A Beneficial Aspect for Patients. Indian J Clin Biochem 2012; 27: 319-321.

https://doi.org/10.1007/s12291-012-0271-2

[16] Patel N. Why is EDTA the anticoagulant of choice for hematology use? Tech Talk, 2009; $7: 1$.

[17] Lillo R, Salinas M, Lopez-Garrigos M. Reducing preanalytical laboratory sample errors through educational and technological interventions. Clin Lab 2012; 58: 911-7.

[18] Lippi G, Becan-McBride K, Behúlová D. Preanalytical quality improvement: in quality we trust; Clin Chem Lab Med 2013; 51: 229-41. https://doi.org/10.1515/cclm-2012-0597

[19] de Baca ME, Gene Gulati G, Kocher W, Schwarting R. Effects of Storage of Blood at Room Temperature on Hematologic Parameters Measured on Sysmex XE-2100. Lab Med 2009; 37: 28-36. https://doi.org/10.1309/1EERK1M02QFJRX6P

[20] Vogelaar SA, Posthuma D, Boomsma D, Kluft C. Blood sample stability at room temperature for counting red and white blood cells and platelets. Vascul Pharmacol 2002; 39: 123-125. https://doi.org/10.1016/S1537-1891(02)00298-7 